\title{
Bilayer Quantum Hall Ferromagnet in a Periodic Potential
}

\author{
Jianmin Sun, ${ }^{1}$ Ganpathy Murthy, ${ }^{2}$ H.A. Fertig, ${ }^{1}$ and Noah Bray-Ali ${ }^{2}$ \\ ${ }^{1}$ Department of Physics, Indiana University, Bloomington, IN \\ ${ }^{2}$ Department of Physics and Astronomy, University of Kentucky, Lexington KY 40506-0055
}

(Dated: October 29, 2018)

\begin{abstract}
The bilayer quantum Hall system at a total filling of $\nu_{T}=1$ has long resisted explanation in terms of a true counterflow superfluid, though many experimental features can be seen to be "almost" that of a superfluid. It is widely believed that quenched disorder is the root cause of this puzzle. Here we model the nonperturbative effects of disorder by investigating the $\nu=1$ bilayer in a strong periodic potential. Our model assumes that fermions are gapped and real spins are fully polarized, and concentrates on the pseudospin variable (the layer index), with the external potential coupling to the topological (Pontryagin) density of the pseudospin. We find that as the potential strength increases, there are ground state transitions in which the topological content of the pseudospin configuration changes. These transitions are generically weakly first-order, with a new quadratically dispersing mode (in addition to the linearly dispersing Goldstone mode) sometimes becoming nearly gapless near the transition. We show that this leads to strong suppressions of both the Kosterlitz-Thouless transition temperature and the interlayer tunneling strength, which we treat perturbatively. We discuss how these results might extend to the case of true disorder.
\end{abstract}

PACS numbers: 73.50.Jt

\section{INTRODUCTION}

It has long been expected theoretically that bilayers offer the possibility of Bose condensation of interlayer excitons [1]. While investigations in electron-hole bilayers are ongoing [2], the electron bilayer system in a large magnetic field at total electron filling $\nu=1$ offers a different way of realizing this goal [3]. There are two important length scales in this system, the interlayer separation $d$ and the magnetic length $l=\sqrt{\frac{\hbar}{e B}}$, (MKS units) where $e$ is the magnitude of the electron charge and $B$ is the magnetic field. In addition to the dimensionless tuning parameter $d / l$, there may also be a layer imbalance $\nu_{\uparrow}-\nu_{\downarrow}$, where $\uparrow, \downarrow$ refer to the top and bottom layer, and an in-plane field $B_{\|}$. The discussion in this paper will be restricted to the case of balanced layers with $\nu_{\uparrow}=\nu_{\downarrow}=\frac{1}{2}$ and $B_{\|}=0$. In this case, by a particle-hole transformation in one layer, one can immediately see the possibility of exciton formation and condensation for small $d / l$.

The first theoretical works on this system two decades ago predicted in the clean limit that at $T=0$ and small $d / l$, the system could be thought of either as a exciton Bose condensate or as a pseudospin quantum Hall ferromagnet [4] with a planar anisotropy. The presence of a gapless, linearly dispersing, Goldstone mode (in the absence of interlayer tunneling) was noted [5] indicating a spontaneous broken symmetry, as was the expectation that when the interlayer tunneling amplitude $h$ became nonzero but small compared to the other energy scales, there would be a delta-function peak in the interlayer conductance $G_{I L}$ at zero bias [6]. Upon the application of an in-plane field $B_{\|}$, the peak is expected to split into two symmetric peaks separated by a bias voltage proportional to $B_{\|}[7]$. Furthermore, the tunneling is expected to occur within a Josephson length $\left(l_{J}=\sqrt{\frac{J}{h}}\right.$, with $J$ being the pseudospin stiffness) of the contacts [6, 7]. Finally, for $h=0$, a Berezinskii-Kosterlitz-Thouless transition [8, 9] at a nonzero temperature $T_{K T}$ is expected, and the counterflow conductance, where current flows in opposite directions in the two layers, is expected to be infinite for $T<T_{K T}$ 7]. An excellent review summarizes the theoretical expectations in the clean system[10].

Experimentally, it is found that as $d / l$ varied, there is a transition [11] between a compressible phase with unquantized Hall conductance for large $d / l$ (presumably adiabatically connected to a system with two decoupled $\nu=\frac{1}{2}$ Composite Fermion [12] Fermi seas [13] in the two layers) and an incompressible phase for $d / l \leq 1.6$ with a quantized Hall conductance.

Where the experimental results deviate from theoretical expectations is in the interlayer tunneling [14 -16] and counterflow 17] properties of the incompressible phase. It is never possible in an experimental sample to make $h$ precisely zero, but it can be made much smaller (of order $100 \mu K$ ) than any other energy scale, including the temperature. In such samples, there is a peak at zero-bias in $G_{I L}$, but its width remains nonzero even at the lowest temperatures 14. Upon the application of an in-plane field, features at nonzero bias are seen to vary in a linear manner with $B_{\|}$, but the peak at zero-bias remains [16], contrary to theoretical expectations, and retains most of the weight. The counterflow conductivity increases as $T$ decreases, but remains finite down to the lowest measured $T$ [17]. Contrary to theoretical expectations, interlayer tunneling occurs throughout the area of the sample [18] rather than within a Josephson length of the contacts. Recently, critical interlayer tunneling currents have been measured [19] (with some puzzling aspects 20]), and are also found to be proportional to the area of the sample. Finally, while a sharp drop in the peak value of the interlayer conductance is seen at a particular $T$, the phe- 
nomenology of this nonzero-temperature transition 21] (if indeed it is a thermodynamic transition) does not seem to be Berezinskii-Kosterlitz-Thouless-like.

Much theoretical work [5 7, 22 43] has been carried out on this system in the past two decades in several directions. We will not attempt to review the vast literature, except to comment that no satisfactory fundamental explanation of the entire spectrum of discrepancies noted above has been proposed yet. It has been recognized that an extra mechanism of dissipation seems to be active in this system, as seen in the finite value of the counterflow conductivity [17], and in the nonzero width of the zero-bias peak in $G_{I L}$. Assuming such a mechanism phenomenologically, several authors have successfully described some fraction of the experimental results, as for example in Ref. [24]. Recent efforts have focused on the effects of quenched disorder 30, 31, 33, 36, 37, 39 42], and succeeded in explaining some aspects of the experiments. Theoretical evidence from studies of critical counterflow 32 and tunneling currents 43 also lead to the identification of quenched disorder as the root cause of the discrepancies between theory and experiment.

In this paper we will start with the assumption that quenched disorder is responsible for the qualitative differences between theory in the clean limit and experiment. As Efros [4] showed long ago, in any incompressible system, disorder necessarily has a nonperturbative effect, because the clean system cannot screen linearly. In twodimensional electron gases, there is the further feature that the fluctuations of the disorder potential, created by positional flutuations of the remote dopants, diverge at long distances. In the semiclassical Efros picture, the quantum Hall system screens by forming compressible puddles (of typical size $s$, the distance between the 2DEG and the dopant layer) separated by incompressible strips of width a few magnetic lengths. This picture is supported by imaging experiments 45 . Based on this picture of strong smooth disorder, two of the present authors previously presented[39] a classical coherence network model displaying some of the experimental phenomenology, such as the proportionality of the interlayer tunneling conductance to the area [18] rather than length of the contacts. A more detailed, though still classical, calculation [42] has recently used the coherence network model to predict a large enhancement of the Josephson length due to disorder.

Here we take a slightly different approach, inspired by studies of the Bose-Hubbard model [46] and one-component quantum Hall systems in a periodic potential [47]. These studies were motivated by trying to develop a field-theoretic understanding of the superfluidMott Insulator transition for bosons or the plateau transition for quantum Hall systems. Note that without a potential, Galilean invariance means that one cannot even obtain a transition in both these cases. Once a secondorder transition has been obtained in the presence of a periodic potential, one may examine the relevance or irrelevance of operators pertaining to various kinds of disorder [4] at this fixed point.

Combining ideas from the Efros picture 44 and the plateau transition work [47], one may hope that a periodic potential will capture the essential nonperturbative features of quenched disorder, and true disorder can be added later [48]. In this paper we will carry out the first part of this program, ending with some speculations regarding true quenched randomness at the end.

As a further simplification, we will assume that fermionic degrees of freedom are gapped out and focus exclusively on the pseudospin degrees of freedom as the only relevant ones. This also involves freezing out the real spin, which may not be quite correct for typical experimental samples [49, 50]. However, recently it has been shown that the interlayer coherent phase is robust to large Zeeman energies, and survives full polarization of real spin [51]. Our study will be of direct relevance to that system, and the qualitative results will likely hold for the low Zeeman energy case as well. From now on, we will refer to "spin" always meaning the pseudospin vector, represented by a unit vector $\mathbf{n}$. An important ingredient is the spin-charge relation [52, 53], which holds in multicomponent systems in a quantum Hall phase [7, 10]. In our context, this states that slow variations of spin contain Coulomb charge:

$$
\delta \rho(\mathbf{r})=\frac{e}{4 \pi} \mathbf{n} \cdot\left(\partial_{x} \mathbf{n} \times \partial_{y} \mathbf{n}\right) .
$$

It is through this $\delta \rho$ that the spins couple to the external potential. For ease of computation we will put our spins on a square lattice, with the lattice spacing chosen to be of the order of the magnetic length $l$. In this case the above, continuum, expression has to be replaced with the spherical area [54] subtended by the three non-coplanar spins $\mathbf{n}_{1}, \mathbf{n}_{2}, \mathbf{n}_{3}$,

$$
\delta Q_{123}=\frac{e}{2 \pi} \tan ^{-1}\left(\frac{\mathbf{n}_{1} \cdot \mathbf{n}_{2} \times \mathbf{n}_{3}}{1+\mathbf{n}_{1} \cdot \mathbf{n}_{2}+\mathbf{n}_{2} \cdot \mathbf{n}_{3}+\mathbf{n}_{3} \cdot \mathbf{n}_{1}}\right) .
$$

The Hamiltonian of our model has the form

$$
\begin{aligned}
\mathcal{H}=- & J \sum_{\left\langle\mathbf{r r}^{\prime}\right\rangle}\left(n_{x}(\mathbf{r}) n_{x}\left(\mathbf{r}^{\prime}\right)+n_{y}(\mathbf{r}) n_{y}\left(\mathbf{r}^{\prime}\right)\right)+\frac{\Gamma}{2} \sum_{\mathbf{r}}\left(n_{z}(\mathbf{r})\right)^{2} \\
& -h \sum_{\mathbf{r}} n_{x}(\mathbf{r})-V_{0} \sum_{\square} f(X, Y) \delta Q_{\square}+H_{U}\left[\delta Q^{2}\right] .
\end{aligned}
$$

Here $J$ is the nearest-neighbor ferromagnetic coupling, and is related to the continuum spin stiffness, $\Gamma$ is the interlayer charging energy, $h$ is the interlayer tunneling amplitude, and $V_{0}$ is the strength of the periodic potential with $f(X, Y)$ being its specific functional form, defined on dual lattice sites $\mathbf{R}=(X, Y)$. The sum in the third term, $\sum_{\square}$, refers to a sum over plaquettes of the lattice, and we have also introduced a local Hubbard interaction $H_{U}$ to model the energy cost (of order the interaction scale $E_{c}=\frac{e^{2}}{\epsilon l}$ ) of localizing a charge within a distance of $l$. We will specify the precise form of these last two terms in the next section. Most of our results are for 
$J=\Gamma$, with our Hubbard $U$ being about ten times $J$, for different values of $V_{0}$. We also vary the size of the unit cell between $12 \times 12$ and $16 \times 16$ lattice sites, with each representing an area of order $100-300 l^{2} \approx s^{2}$ ( $s$ is the distance between the 2DEG and the dopant layer), consistent with values of the expected size of Efros puddles for relevant experimental samples.

Our results, which we summarize here, and elaborate upon in later sections, show that there are some surprising features present in this model. Consider first $h=0$. We find a sequence of ground state transitions where the topological content of the spin configuration changes. For sufficiently large $U$, the first transition is second-order while the others are generically weakly first-order. There is always a linearly dispersing Goldstone mode (which we will henceforth call the $G$-mode) indicating the broken $U(1)$ symmetry for $h=0$. The next higher mode is usually quadratically dispersing (we will call it the $Q$-mode), with a gap that sometimes becomes very small near the transitions. In the case when the transition is continuous, the gap of the $Q$-mode vanishes at the transition continuously. It is important to note that true disorder will generically convert first-order transitions to secondorder [56], and thus the $Q$-mode is expected to be truly gapless at the ground state transitions in the disordered model. Two distinct effects can be traced to the transitions and the properties of the $Q$-mode near them. At values of $V_{0}$ near the transitions, there are configurations close in energy with a different topological density, which means that the system becomes highly polarizable. In an effective $X Y$ model (to be described in Section) this leads to a greatly reduced core energy for vortices, or a greatly increased fugacity, which strongly suppresses the KTtransition temperature. Secondly, when the $Q$-mode gap is small, thermal fluctuations of the $Q$-mode are present at realistic temperatures, and strongly suppress the interlayer tunneling amplitude $h$. Finally, the low-lying $Q$-excitations offer one possible microscopic mechanism for the pervasive and puzzling dissipation seen in experiments. These are the main results of our work.

Let us remark briefly about the realistic case $h \neq 0$ but smaller than any other energy scale in the problem, including the temperature. The ground state phase transitions are controlled by the competition between the core energies of merons/antimerons and the external potential. Both these are on the scale of $E_{c}$. Therefore, we expect a nonzero but tiny $h \ll T, V, E_{c}$ will not affect the transitions in any qualitative way. Similarly, for the collective mode dispersions, the primary effect of a tiny $h$ is to gap the $G$-mode at $q=0$. We expect all other modes to be robust against the introduction of a small $h$.

It is important to note that in the clean system, $h$ is relevant below $T_{K T}[9]$, and the regimes of small and large $h$ are adiabatically connected to each other, with no phase transition separating them. This is not true for the system with a periodic potential or strong, smooth disorder. As $h$ is increased while all other parameters (such as $E_{c}$ and $V$ ) are kept fixed, the system will undergo ground state phase transitions in which the topological density reduces, presumably becoming the uniform ferromagnetic state for very large $h \gg V$. This qualitative distinction between weak and strong tunneling in the presence of a periodic potential (and presumably a strong smooth disorder potential) is consistent with experiments, which see the almost-superfluid phenomenology only for weak $h$.

The plan of this paper is as follows: In Section II we will present the precise definition of our model and present sample ground-state configurations. Section III presents our method for computing the collective mode spectra of excitations above the ground-state, and discusses the effects of the potential strength $V$, the inter-layer tunneling $h$, and the interaction strength $U$ on this. This is followed by a discussion of how one may extract the spin stiffness of our model from the numerical results. Section IV discusses two non-trivial fluctuation effects in this system, first how the quadratically dispersing collective mode can suppress inter-layer tunneling, and then the suppression of $T_{K T}$ near quantum phase transitions due to vortex excitations. We end with some conclusions, caveats, and speculations on what our studies tell us about the case of true disorder in Section V.

\section{MODEL AND GROUND-STATES}

The form of the Hamiltonian of our model, Eq. (3), is guided by the following considerations: (i) We ignore fermionic excitations at the very outset, since in any incompressible state, the energy to create a fermion is of the order of the Coulomb scale $E_{c}$, which is much larger than the other relevant energy scales. Similarly we ignore the dynamics of the real spin, assuming that it is fully polarized [51]. (ii) We put the system on a lattice for computational convenience 28. As long as all the relevant length scales, such as the size of the puddles, are much larger than the chosen lattice scale, we expect that our results will be physically correct. To be specific, we will choose the lattice spacing $a=l \sqrt{2 \pi}$ such that each elementary plaquette has the area of one cyclotron orbit, and at $\nu_{T}=1$, has one electron. (iii) In the continuum, when there is no external potential, the Hamiltonian should reduce to the continuum form[7] in the long-wavelength limit. (iv) The coupling of the external potential to the spin (i.e., layer) degrees of freedom must be through the lattice generalization [54] of the topological charge 52, 53]. (v) To account for short-distance effects beyond the spin stiffness, we introduce a Hubbard on-plaquette interaction term. (vi) Finally, we ignore the long distance component of the Coulomb interaction between induced charges for computational convenience, assuming that the Hubbard term can capture all qualitative effects.

We are now ready to make the form of the Hamiltonian 
precise. We consider a square lattice of points labelled by $\mathbf{r}$, with the corresponding dual lattice labelled by $\mathbf{R}$, with the convention that the site $\mathbf{R}$ corresponding to $\mathbf{r}$ lies half a lattice unit to the right and above $\mathbf{r}$. The external potential naturally lives on the dual lattice. Each dual site $\mathbf{R}$ defines a plaquette, whose sites can be labelled $\mathbf{r}_{1}, \mathbf{r}_{2}, \mathbf{r}_{3}, \mathbf{r}_{4}$, starting from $\mathbf{r}=\mathbf{r}_{1}$ and going counterclockwise. The topological charge corresponding to a given triplet of spins was given above in Eq. (2). The charge in a plaquette is defined as

$$
\delta Q_{\square}(\mathbf{R})=\delta Q_{123}+\delta Q_{134}=\delta Q_{124}+\delta Q_{234} .
$$

To fully specify the third term on the RHS of Eq. (3) we need the functional form of $f(X, Y)$. We choose the simplest possible periodic form:

$$
f(X, Y)=\sin \left(\frac{2 \pi X}{N a}\right) \sin \left(\frac{2 \pi Y}{N a}\right) .
$$

$N a$ is the size of the unit cell, which contains four puddles. We have experimented with other forms and observed no qualitative differences. Finally, we turn to the last term of Eq. (3). In order to eliminate spurious configurations with equal and opposite topological charges in triplets 123 and 134 but no net charge in the plaquette, we write the Hubbard term as the symmetrized sum of all the triplets in a plaquette:

$H_{U}=\frac{U}{4} \sum_{\mathbf{R}}\left(\left(\delta Q_{123}\right)^{2}+\left(\delta Q_{134}\right)^{2}+\left(\delta Q_{124}\right)^{2}+\left(\delta Q_{234}\right)^{2}\right)$.

Hartree-Fock calculations are a reasonable starting point for estimating the values of the various parameters entering the Hamiltonian. $J$ and $\Gamma$ are of the order of a few percent of $E_{c}$ in such calculations, while $U$ should be of the order of $E_{c}$, being the energy to localize a charge of order $e$ within a length scale $l$. We will typically assume $J=\Gamma$, and $U$ between 8 and 20 times $J$. Most of our results are for the size of the unit cell being $N=16$, but we will show results for $N=12$ as well. These values are realistic given $l \approx 200 \AA$ and the distance to the dopant layer $s \approx 2000 \AA$.

We parameterize the spins by $z(\mathbf{r}) \equiv n_{z}(\mathbf{r})$ and $\phi(\mathbf{r})$ which is the $X Y$ angle $\left[n_{x}(\mathbf{r})=\right.$ $\left.\sqrt{1-z(\mathbf{r})^{2}} \cos \phi(\mathbf{r}), \quad n_{y}(\mathbf{r})=\sqrt{1-z(\mathbf{r})^{2}} \sin \phi(\mathbf{r})\right]$. We find the ground state configurations by starting with a random seed configuration, followed by simulated annealing. Several different random seeds were tried at every value of $V_{0}$ to eliminate the possibility of settling into a metastable minimum.

We begin by showing the ground state spin configuration for a $16 \times 16$ unit cell at $V=3$ and $U=8$ (in units of $J=\Gamma=1$ ). As shown in Fig. (11), there is a vortex/antivortex at the center of each puddle, partially screening the external potential. The length of the arrow indicates the projection of the spin in the $x y$-plane, while the color indicates whether the spin points in the up or down direction in $z$. A different way to plot the same

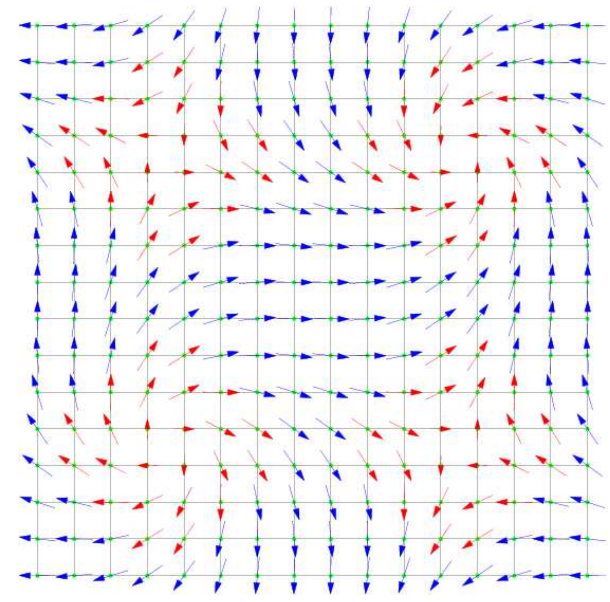

FIG. 1: (Color online) The ground state configuration for a $16 \times 16$ unit cell with the strength of the periodic potential (in units where $J=1$ ) being $V=3.0$, and the Hubbard interaction is $U=8.0$. The lengths of the spins denote their planar projection. Note a vortex/antivortex at the center of each puddle.
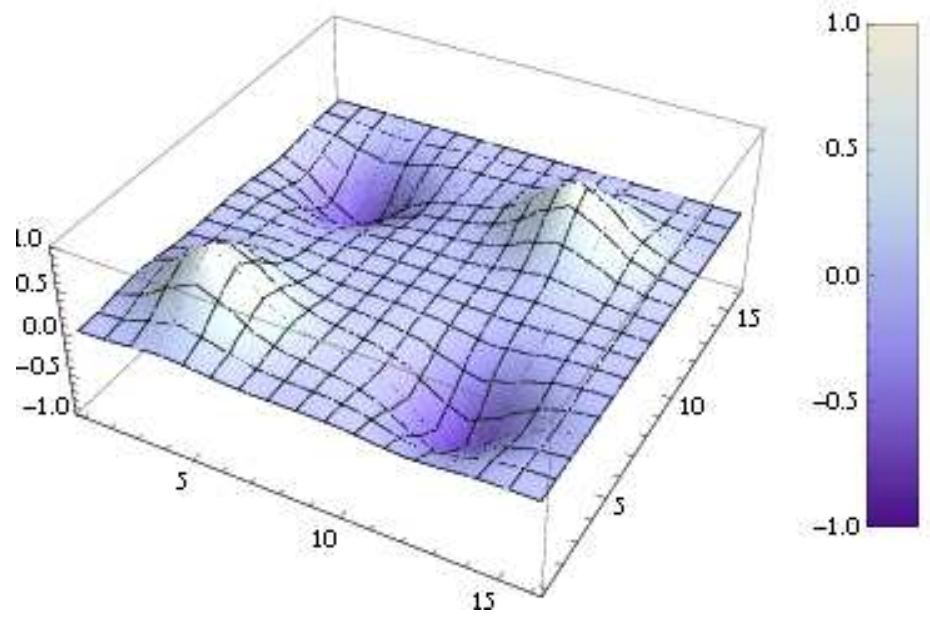

FIG. 2: (Color online)The topological density of the spins in the ground state configuration for a $16 \times 16$ unit cell with $V=3.0$, and $U=8.0$ shown as a three-dimensional plot.

configuration is to look instead at the topological density of the spins. This is shown as a three-dimensional plot in Fig. (2). A more complicated configuration is shown in Fig. (3), where $V$ has now been increased to 7 . Each puddle now has two vortex-antivortex pairs. The corresponding topological density is plotted as a countour plot in Fig. (4). The phase transitions correspond to values of $V$ where the ground state energy of one configuration becomes lower than another. The very first of these transitions (for sufficiently large $U$ ) is second-order. For small $V$ the ground state is uniform and ferromagnetic as at $V=0$. At a critical $V=V_{c}$ (which happens to be $V_{c}=4.2$ for $U=18$ ) there is an instability of the ground state, which, when followed, leads to a ground 


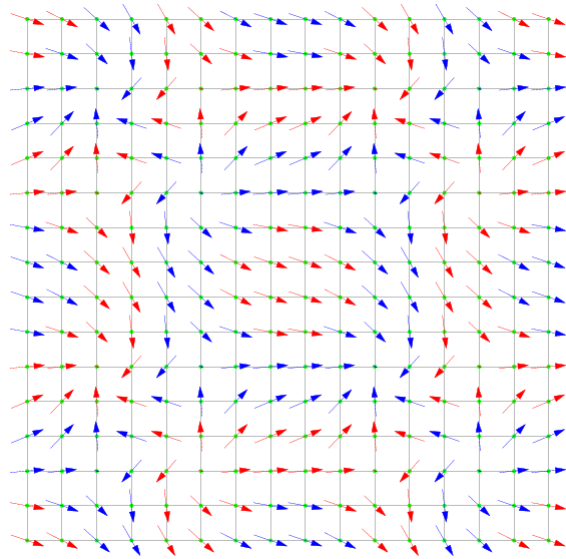

FIG. 3: (Color online) The ground state configuration for $V=7.0$ and $U=8.0$.

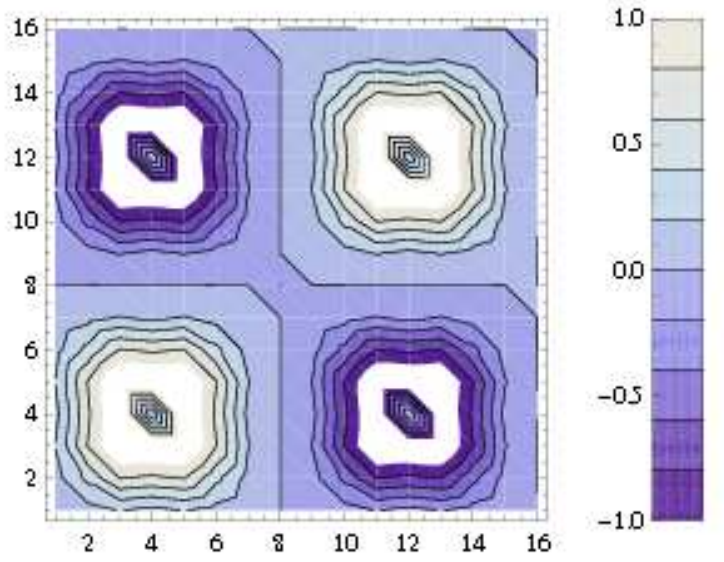

FIG. 4: (Color online) The topological charge in the ground state configuration for $V=7.0$ and $U=8.0$.

state with a nonzero topological density. While the very first of these transitions is second-order, the rest seem to generically be weakly first-order. The ground state energies cross with different slopes as functions of $V$.

In Fig. (5) we show the ground state of the $12 \times 12$ unit cell at $V=3$ and $U=8$. The presence of two vortexantivortex pairs is evident, as is the qualitative similarity to the case of the $16 \times 16$ unit cell of Fig. (1). Generally we find that all the qualitative features of the different ground states are the same for different sizes of the unit cell, but the specific values at which the transitions happen do depend on unit cell size.

\section{DEVIATIONS FROM THE GROUND STATE}

Having obtained classical groundstates of this system, we are in a position to consider the energetics and dynamics associated with deviations from it. In what follows we will focus on small deviations, such that the system can

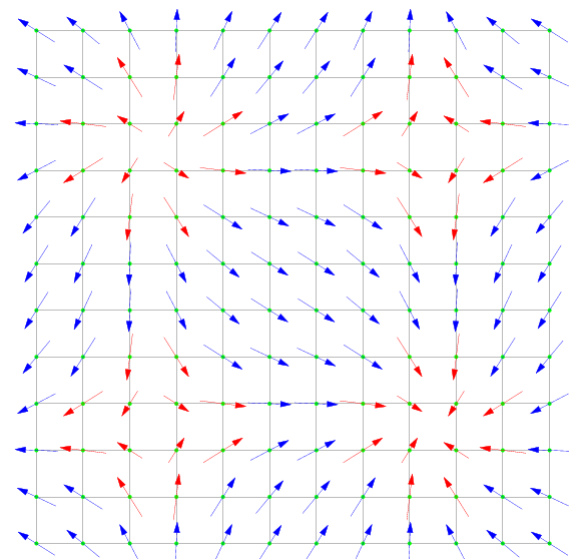

FIG. 5: (Color online) The configuration of the $12 \times 12$ lattice at $V=3$ and $U=8$. Comparing to Fig. (1) we see that the presence of the vortex-antivortex pairs is independent of the size of the unit cell, though the values of critical $V$ at which the ground state transitions take place are different.

be analyzed within a quadratic theory. Two types of quantities are of particular interest, the collective modes of the system, and the effective spin stiffness. The first of these can be used to explore the effects of thermal fluctuations, and the second is important in determining the effects of thermally generated vortices (i.e., KosterlitzThouless physics.)

\section{A. Collective Modes}

Once a periodic ground state configuration $\bar{z}(\mathbf{r}), \bar{\phi}(\mathbf{r})$ has been found, one can look at collective mode dynamics around this state. For dynamics, one needs the full action, which is

$$
S=S_{B}-\int d t \mathcal{H}
$$

where $S_{B}$, the Berry phase term [55], measures the spherical area covered by a closed path followed by a spin in time,

$$
S_{B}=\frac{\hbar}{2} \int d t \int_{0}^{1} d \xi \sum_{\mathbf{r}} \mathbf{n}(\mathbf{r}, t, \xi) \cdot \partial_{t} \mathbf{n}(\mathbf{r}, t, \xi) \times \partial_{\xi} \mathbf{n}(\mathbf{r}, t, \xi) .
$$

Here $\xi$ is an auxiliary variable and $\mathbf{n}(\mathbf{r}, t, \xi)$ is chosen such that at $\mathbf{n}(\mathbf{r}, t, 0)=\hat{e}_{z}$ and $\mathbf{n}(\mathbf{r}, t, 1) \equiv \mathbf{n}(t, \mathbf{r})[22$. Also, we have made the $\hbar$ explicit, and assumed that the spin is $\frac{1}{2}$. This is reasonable since the underlying fermionic system has one electron per cyclotron orbit; with our choice of lattice spacing this translates to one electron per site.

The Berry phase term may be conveniently expressed as

$$
S_{B}=\int d t \frac{1}{2} \sum_{\mathbf{r}}(z(\mathbf{r}) \dot{\phi}(\mathbf{r})-\phi(\mathbf{r}) \dot{z}(\mathbf{r})) .
$$


Following standard methods, and recalling that any variation of $\mathbf{n}$ has to be perpendicular to $\mathbf{n}$ we obtain the equation of motion

$$
\partial_{t} \mathbf{n}=\mathbf{n} \times \frac{\delta \mathcal{H}}{\delta \mathbf{n}} .
$$

The ground state configuration is static, and satisfies

$$
\left.\frac{\partial \mathcal{H}}{\partial z(\mathbf{r})}\right|_{\bar{z}, \bar{\phi}}=\left.\frac{\partial \mathcal{H}}{\partial \phi(\mathbf{r})}\right|_{\bar{z}, \bar{\phi}}=0 .
$$

Expanding in small fluctuations around the ground state,

$$
z(\mathbf{r})=\bar{z}(\mathbf{r})+\zeta(\mathbf{r}), \quad \phi(\mathbf{r})=\bar{\phi}(\mathbf{r})+\varphi(\mathbf{r}),
$$

we obtain the linearized equations of motion

$$
\begin{aligned}
& \dot{\zeta}(\mathbf{r})=-\frac{\partial^{2} \mathcal{H}}{\partial \phi(\mathbf{r}) \partial z\left(\mathbf{r}^{\prime}\right)} \zeta\left(\mathbf{r}^{\prime}\right)-\frac{\partial^{2} \mathcal{H}}{\partial \phi(\mathbf{r}) \partial \phi\left(\mathbf{r}^{\prime}\right)} \varphi\left(\mathbf{r}^{\prime}\right) \\
& \dot{\varphi}(\mathbf{r})=\frac{\partial^{2} \mathcal{H}}{\partial z(\mathbf{r}) \partial z\left(\mathbf{r}^{\prime}\right)} \zeta\left(\mathbf{r}^{\prime}\right)+\frac{\partial^{2} \mathcal{H}}{\partial z(\mathbf{r}) \partial \phi\left(\mathbf{r}^{\prime}\right)} \varphi\left(\mathbf{r}^{\prime}\right) .
\end{aligned}
$$

As can be seen, the Hessian matrix of second derivatives (computed at the ground state configuration) appears on the right hand side,

$$
H_{s s^{\prime}}\left(\mathbf{r}, \mathbf{r}^{\prime}\right)=\frac{\partial^{2} \mathcal{H}}{\partial w(\mathbf{r}, s) \partial w\left(\mathbf{r}^{\prime}, s^{\prime}\right)},
$$

where we have introduced indices $s, s^{\prime}$ taking the values 1,2 for $\zeta$ and $\varphi$ respectively, thereby organizing $\zeta(\mathbf{r})=w(\mathbf{r}, 1), \varphi(\mathbf{r})=w(\mathbf{r}, 2)$ into a two-component vector. It is important to note that for $h=0$ one can rotate all the $\phi$ by the same amount producing no change in energy, which means that the Hessian matrix $H$ has an eigenvector with a zero eigenvalue.

The next step is to exploit the larger unit cell and partially diagonalize the system in terms of a wave-vector $\mathbf{k}$ lying in the Brillouin Zone (BZ), arranging the $2 N^{2}$ variables in a unit cell into a single vector. We can now define the $2 N^{2} \times 2 N^{2}$ matrices

$$
\begin{aligned}
\tilde{H}_{s s^{\prime}}\left(\mathbf{k}: \mathbf{r}, \mathbf{r}^{\prime}\right) & =e^{-i \mathbf{k} \cdot\left(\mathbf{r}-\mathbf{r}^{\prime}\right)} H_{s s^{\prime}}\left(\mathbf{r}, \mathbf{r}^{\prime}\right), \\
K_{s s^{\prime}}\left(\mathbf{r}, \mathbf{r}^{\prime}\right) & =\left(\sigma_{2}\right)_{s s^{\prime}} \otimes \delta_{\mathbf{r}, \mathbf{r}^{\prime}},
\end{aligned}
$$

where $\sigma_{2}$ is a Pauli matrix. It is henceforth understood that in all matrices $\mathbf{r}, \quad \mathbf{r}^{\prime}$ are restricted to a single unit cell. Note that both matrices are Hermitian, but differ under transposition:

$$
\begin{aligned}
(\tilde{H}(\mathbf{k}))^{T} & =\tilde{H}(-\mathbf{k}) \\
K^{T} & =-K .
\end{aligned}
$$

The earlier condition on the Hessian having an eigenvector with zero eigenvalue translates to the vanishing of the smallest eigenvalue of $\tilde{H}$ as $\mathbf{k} \rightarrow 0$. This will be important for the computation of the spin stiffness.

Now the equations of motion can be written in a compact form

$$
\omega K \psi^{[R]}(\mathbf{k})=\tilde{H}(\mathbf{k}) \psi^{[R]}(\mathbf{k}) .
$$

This is a generalized eigenvalue problem with both $K$ and $\tilde{H}$ being Hermitian. We first solve for the eigenvalues and eigenvectors of the auxiliary problem

$$
K \tilde{H} \psi_{\lambda}^{[R]}(\mathbf{k})=\omega_{\lambda}(\mathbf{k}) \psi_{\lambda}^{[R]}(\mathbf{k})
$$

to get the right eigenvectors $\psi_{\lambda}^{[R]}$. Taking the Hermitian adjoint and carrying out a few manipulations, one obtains the left eigenvectors

$$
\left(\psi_{\lambda}^{[R]}(\mathbf{k})\right)^{\dagger} K=\psi_{\lambda}^{[L]}(\mathbf{k}) .
$$

Using the hermiticity of $K$ and $\tilde{H}$, and Eqs. (17) and (20) it is easy to show that the eigenvalues occur in pairs with equal magnitude and opposite sign.

The normalization condition on the eigenvectors can be chosen to be

$$
\left(\psi_{\lambda}^{[R]}(\mathbf{k})\right)^{\dagger} K \psi_{\lambda^{\prime}}^{[R]}(\mathbf{k})=\operatorname{sgn}\left(\omega_{\lambda}(\mathbf{k})\right) \delta_{\lambda \lambda^{\prime}},
$$

where the $\operatorname{sgn}\left(\omega_{\lambda}\right)$ is essential because $K$ is not positive definite. Now, as a consequence of Eqs. (19) and (21) the eigenvectors also satisfy

$$
\left(\psi_{\lambda}^{[R]}(\mathbf{k})\right)^{\dagger} \tilde{H} \psi_{\lambda^{\prime}}^{[R]}(\mathbf{k})=\left|\omega_{\lambda}(\mathbf{k})\right| \delta_{\lambda \lambda^{\prime}} .
$$

Henceforth we will use only the right eigenvectors, dropping the superscript $[R]$.

We find the following results for the collective mode dispersions as functions of $V$. Since we set the interlayer tunneling $h=0$, there is always a gapless, linearly dispersing, Goldstone mode arising from the breaking of the continuous $U(1)$ global symmetry. We will henceforth call this the $G$-mode. The $G$-mode is generically the lowest mode throughout the BZ, but may not be so close to a transition. The next higher energy mode is quadratically dispersing at $\mathbf{q}=0$, and will play an important role in what follows. We will henceforth call it the $Q$-mode. At the first transition, which is second-order for large $U$, the gap of this mode vanishes at $\mathbf{q}=0$. In Fig. (6) we show the dispersions of the two lowest energy modes at $V=4.2$ and $U=24$, close to the transition. It is clear that the $Q$-mode is the lowest energy mode for a substantial part of the BZ.

At a generic transition, however, the gap of the $Q$ mode at $\mathbf{q}=0$ does not vanish. Summarizing the behavior of the $Q$-mode over the entire range of $V$ we investigated, we present Fig. (7), which shows the $Q$-mode gap as a function of $V$ for $U=8$. We also examined the "wave functions" of the $Q$-mode near the transitions. The $\phi$-component appears to be strongly peaked at the vortex/antivortex cores, while the $z$-component is more broadly distributed. Roughly speaking this may be understood in terms of motion of the merons around their minimum potential sites, with a very flat effective curvature, indicating that "room" for further merons is developing in the potential well. (Similar behavior has 


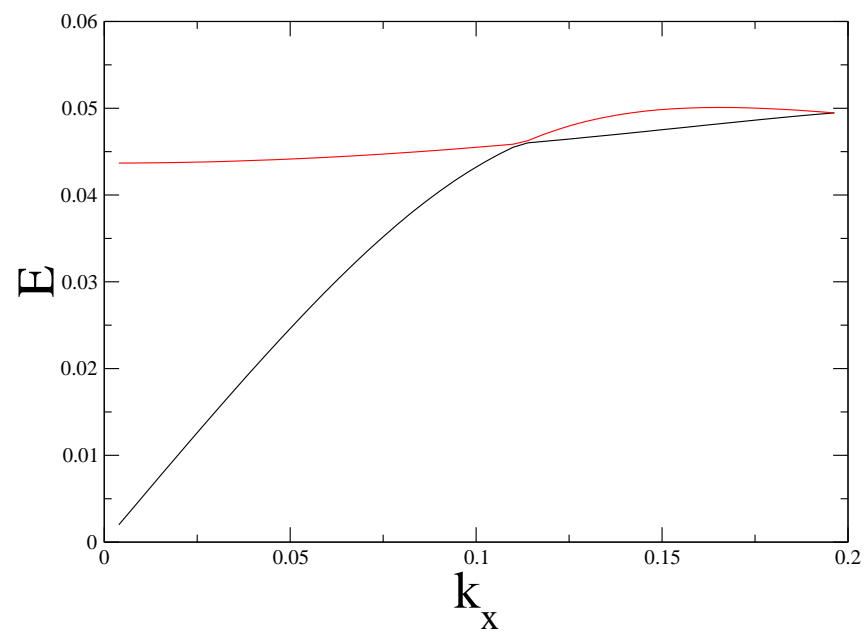

FIG. 6: (Color online) The two lowest collective modes for $V=4.3$ and $U=18$. This is just before the transition from the uniform ferromagnetic state to a striped state, and is generically second-order at sufficiently large $U$. Note the almost gapless quadratically dispersing mode.

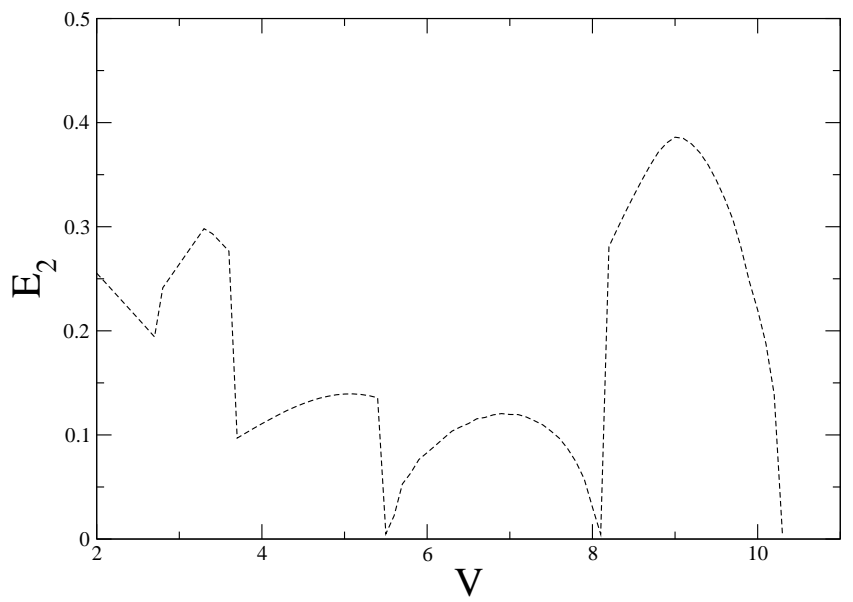

FIG. 7: The gap at $\mathbf{q}=0$ of the $Q$-mode as a function of $V$. Note that it does not generically vanish at a transition, though there are transitions where it becomes particularly small.

been observed in simulations of this system using an $X Y$ model [30].) Moreover, the $Q$-mode dispersion is typically quite flat, indicating that these are fairly welllocalized excitations. Another interesting point to note, shown in Fig. (8), is that there are large fluctuations of the $G$-mode velocity as $V$ changes. It should be kept in mind that these are results to quadratic order in the fluctuations around the ground state configuration. Interactions between the $G$ - and $Q$ - modes could affect the gap of the $Q$-mode and the spin-wave velocity as well.

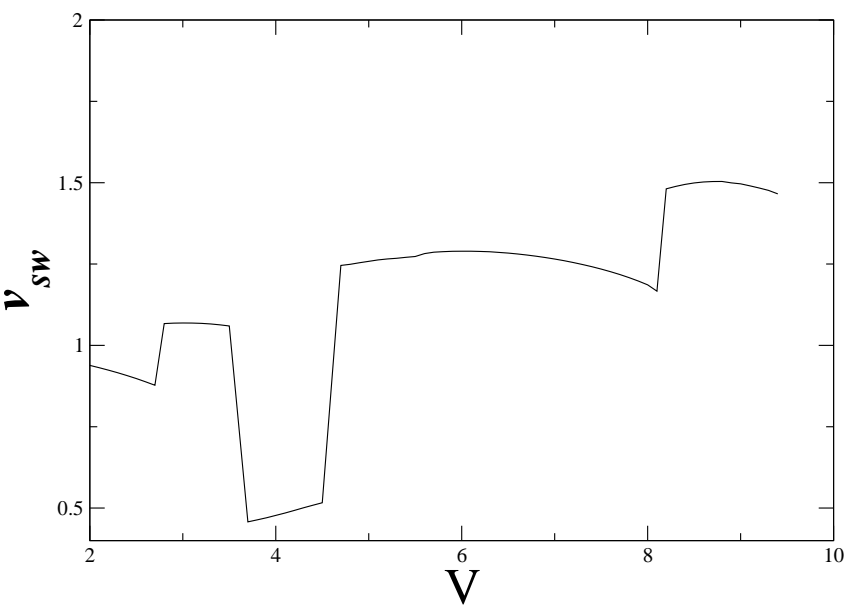

FIG. 8: The spinwave velocity of the $G$-mode as a function of $V$. Note that it never vanishes, but that it can vary by a factor of three, and have significant discontinuities at transitions.

\section{B. Ground-State Spin Stiffness}

Another related quantity of interest for this system is the spin stiffness, which determines among other things the interactions between highly separated vortexantivortex pairs. We begin by describing how it may be computed for an arbitrary ground state configuration. We define the spin stiffness by calculating the energy $U$ for a particular spatially dependent configuration of $\varphi$ and comparing it to the reference energy $U_{\text {cont }}$ of the same configuration in a free continuum model,

$$
U_{\text {cont }}=\frac{1}{2} \mathcal{K}_{s} \int d^{2} \mathbf{r}(\nabla \phi)^{2}
$$

The least expensive way to twist the system is controlled by the smallest eigenvalue of the Hessian $\tilde{H}(\mathbf{k})$ as $|\mathbf{k}| \rightarrow$ 0 . Note that these eigenvalues are not the same as the energies of the eigenmodes found in Eq. (19), which are the solutions to the dynamical problem. We consider a sample with lengths $L_{x}, L_{y}$ in the $x$ and $y$ directions respectively. We denote the eigenvalues of $\tilde{H}(\mathbf{k})$ as $\epsilon_{n}(\mathbf{k})$ and the corresponding eigenvectors as $\chi_{n}(\mathbf{k}, \mathbf{r}, s)$. (Here $s=1,2$ correspond respectively to fluctuations of $\zeta$ and $\varphi$.) Since $\tilde{H}$ is Hermitian and represents a stable ground state, the eigenvalues are all positive semidefinite, and the eigenvectors are normalized in the usual way,

$$
\left(\chi_{n}(\mathbf{k})\right)^{\dagger} \chi_{n^{\prime}}(\mathbf{k})=\delta_{n n^{\prime}}
$$

We can express a generic fluctuation $\varphi$ of the phase field $\phi(\mathbf{r})$ from the ground state at any $\mathbf{r}$ inside the unit cell in the presence of a unit amplitude of the lowest eigenvector, which we call $\chi_{0}(\mathbf{k})$, as

$$
\varphi(\mathbf{r})=\frac{1}{\sqrt{N_{c}}} \sum_{\mathbf{k}} \sum_{n} e^{i \mathbf{k} \cdot \mathbf{r}} p_{n}(\mathbf{k}) \chi_{n}(\mathbf{k}, \mathbf{r}, 2),
$$

where $N_{c}=\frac{L_{x} L_{y}}{N^{2} a^{2}}$ is the number of unit cells. The reality of $\zeta, \quad \varphi$ forces $p_{n}^{*}(\mathbf{k})=p_{n}(-\mathbf{k})$ with the convention that 


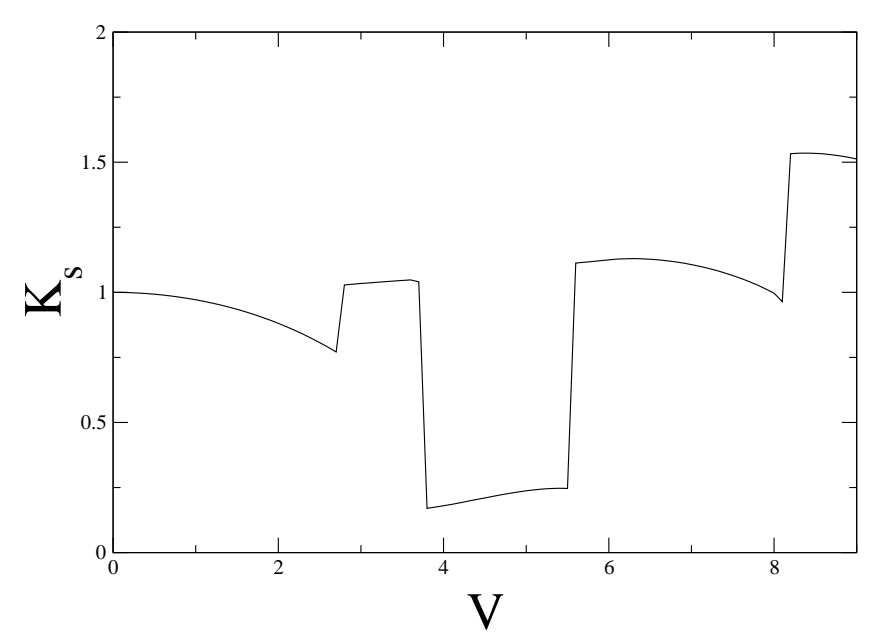

FIG. 9: Spin stiffness as a function of potential strength $V$, normalized to nearest-neighbor ferromagnetic exchange $J$.

$\chi_{n}^{*}(\mathbf{k})=\chi_{n}(-\mathbf{k})$. The energy of such a fluctuation is easily seen to be

$$
U=\sum_{\mathbf{k}, n} \epsilon_{n}(\mathbf{k})\left|p_{n}(\mathbf{k})\right|^{2}
$$

To make the calculation convenient, we choose $\mathbf{k}_{\min }=$ $k_{x, \min } \hat{e}_{x}$ with $k_{x, \min }=\frac{2 \pi}{L_{x}}$ being the minimum nonzero value allowed. Then, bearing in mind the reality of $\varphi$, we can express this as

$$
\varphi(\mathbf{r})=\frac{2}{\sqrt{N_{c}}} p_{0}\left(\mathbf{k}_{\min }\right) \chi_{0}\left(\mathbf{k}_{\min }, \mathbf{r}, 2\right) \sin \left(\frac{2 \pi x}{L_{x}}\right) .
$$

Comparing the energy of Eq. (26) to that of the continuum version Eq. (23), we obtain

$$
\mathcal{K}_{s}=\frac{\epsilon_{0}\left(\mathbf{k}_{\min }\right)}{N^{2} a^{2} k_{\text {min }}^{2}\left|\chi_{0}\left(\mathbf{k}_{\min }, \mathbf{r}=0,2\right)\right|^{2}} .
$$

To be precise, this is the spin stiffness computed at $T=0$, because thermal fluctuations have not been included.

The results for the spin stiffness (Fig. 9) as a function of potential strength $V$ show prononounced jumps. The discontinuities in $\mathcal{K}_{s}$ coincide with transitions in the topological density of the ground-state configuration. Notice that the spin stiffness is generally of the order of $J$, the nearest-neigbor ferromagnetic coupling in Eq. 3. Depending on the topological density in the groundstate configuration, $\mathcal{K}_{s} / J$ can be either significantly enhanced (for example $V / J \leq 8.15$ ) or reduced (for example, $3.75 \leq V / J \leq 5.5)$.

\section{FLUCTUATION EFFECTS}

In a purely quadratic theory, fluctuations will have little effect on most quantities of interest. However, the Hamiltonian of our system contains non-linear couplings which can have important qualitative effects. Already included in our groundstate analysis is the underlying $\mathcal{O}(3)$ nature of the spins, which supports the (charged) merons that are induced by the periodic potential. Thermal fluctuations in which meron-antimeron pairs are generated above the groundstate can spoil the spin stiffness of the system, rendering it dissipative, above an effective Kosterlitz-Thouless (KT) transition temperature. In principle, interlayer tunneling may have even more profound effects, for example removing the possibility of a true thermodynamic KT transition in the clean limit [9, 29]. In this section we describe how fluctuation effects in this model can greatly suppress the impact of interlayer tunneling, and furthermore lead to a lowering of the KT transition temperature, particularly near transitions between different groundstates.

\section{A. Suppression of Interlayer Tunneling by Collective Modes}

We begin with an evaluation of the effective (i.e., renormalized) interlayer tunneling amplitude due to quadratic fluctuations around the ground state. As discussed in the Introduction, this can be greatly depressed by the presence of the $Q$-mode, particularly if the gap is quite small, as sometimes happens in the vicinity of a groundstate transition.

The fluctuations around the ground state can be expanded as

$$
w(\mathbf{r}, s, t)=\frac{1}{\sqrt{N_{c}}} \sum_{\mathbf{k}, \lambda} \tilde{w}_{\lambda}(\mathbf{k}) e^{i\left(\mathbf{k} \cdot \mathbf{r}-\omega_{\lambda} t\right)} \psi_{\lambda}(\mathbf{k}, \mathbf{r}, s)
$$

where $N_{c}$ is the number of unit cells in the lattice and $\tilde{w}_{\lambda}(\mathbf{k})$ is the amplitude of $w$ in the mode $\lambda, \mathbf{k}$. The reality of $w$ means that $\left(\psi_{\lambda}(\mathbf{k})\right)^{*}$ is an eigenvector of $\tilde{H}(-\mathbf{k})$ with eigenvalue $-\omega_{\lambda}(\mathbf{k})$, that is

$$
\begin{aligned}
\left(\psi_{\lambda}(\mathbf{k}, \mathbf{r}, s)\right)^{*} & =\psi_{-\lambda}(-\mathbf{k}, \mathbf{r}, s), \\
\left(\tilde{w}_{\lambda}(\mathbf{k})\right)^{*} & =\tilde{w}_{-\lambda}(-\mathbf{k}) .
\end{aligned}
$$

Now we are ready to re-express the action in terms of these modes. In order to carry out thermal averages, we use the imaginary time path integral

$$
\begin{aligned}
-S= & \int_{0}^{\beta} d t\left(\sum_{\mathbf{r}} \frac{i}{2}[\zeta(\mathbf{r}) \dot{\varphi}(\mathbf{r})-\varphi(\mathbf{r}) \dot{\zeta}(\mathbf{r})]\right. \\
& \left.-\frac{1}{2} \sum_{\mathbf{r r}^{\prime}, s s^{\prime}} w(\mathbf{r}, s) H_{s s^{\prime}}\left(\mathbf{r}, \mathbf{r}^{\prime}\right) w\left(\mathbf{r}^{\prime}, s^{\prime}\right)\right) .
\end{aligned}
$$

When we expand in normal modes, $\tilde{w}$ now acquires a dependence on $i \omega_{n}$ as well:

$$
\begin{gathered}
w(\mathbf{r}, t)=\frac{1}{\beta \sqrt{N_{c}}} \sum_{i \omega_{n}, \mathbf{k}, \lambda} \tilde{w}_{\lambda}\left(\mathbf{k}, i \omega_{n}\right) e^{i\left(\mathbf{k} \cdot \mathbf{r}-\omega_{n} t\right)} \psi_{\lambda}(\mathbf{k}, \mathbf{r})(34) \\
\left(\tilde{w}_{\lambda}\left(\mathbf{k}, i \omega_{n}\right)\right)^{*}=\tilde{w}_{-\lambda}\left(-\mathbf{k},-i \omega_{n}\right) .
\end{gathered}
$$


The last condition means that we can take $\tilde{w}_{\lambda>0}\left(\mathbf{k}, i \omega_{n}\right)$ over the entire BZ and for all $i \omega_{n}$ to be independent, while the $\tilde{w}_{\lambda<0}$ are dependent. Substituting the expansion for $\delta w$ in terms of the normal modes, and using the properties of the wavefunctions Eqs. (21/22131) we obtain

$$
-S=\frac{1}{\beta} \sum_{i \omega_{n}, \mathbf{k}, \lambda>0}\left|\tilde{w}\left(i \omega_{n}, \mathbf{k}, \lambda\right)\right|^{2}\left[i \omega_{n}-\omega_{\lambda}(\mathbf{k})\right] .
$$

As can be seen, the real part of $-S$ is negative definite, as it should be for the convergence of the path integral. This simple form for the action leads to

$$
\left\langle\tilde{w}_{\lambda}\left(\mathbf{k}, i \omega_{n}\right) \tilde{w}_{\lambda^{\prime}}\left(\mathbf{k}^{\prime}, i \omega_{n}^{\prime}\right)\right\rangle=\frac{\delta_{\lambda,-\lambda^{\prime}} \delta_{\mathbf{k},-\mathbf{k}^{\prime}} \delta_{\omega_{n},-\omega_{n}^{\prime}}}{i \omega_{n}-\left|\omega_{\lambda}(\mathbf{k})\right|} .
$$

Now we are ready to find the correlation functions of the fluctuations $w$. The simplest is $\left\langle(w(\mathbf{r}))^{2}\right\rangle$. Clearly, this will be

$$
\left\langle(w(\mathbf{r}, s))^{2}\right\rangle=-\frac{1}{N_{c} \beta} \sum_{i \omega_{n}, \mathbf{k}, \lambda>0} \frac{\left|\psi_{\lambda}(\mathbf{k}, \mathbf{r}, s)\right|^{2}}{i \omega_{n}-\omega_{\lambda}(\mathbf{k})} .
$$

The sum over $i \omega_{n}$ can be done by standard methods to get $n_{\lambda}(\mathbf{k})+\frac{1}{2}$, where $n_{\lambda}(\mathbf{k})=\left(\exp \beta \omega_{\lambda}(\mathbf{k})-1\right)^{-1}$ is the boson occupation function, so that

$$
\left\langle(w(\mathbf{r}, s))^{2}\right\rangle=\frac{1}{N_{c}} \sum_{\mathbf{k}, \lambda>0}\left|\psi_{\lambda}(\mathbf{k}, \mathbf{r}, s)\right|^{2}\left[n_{\lambda}(\mathbf{k})+\frac{1}{2}\right] .
$$

We now consider the effect of these fluctuations on interlayer tunneling. The effect as we shall see is most pronounced where the $Q$-mode gap is small. In our periodic model this occurs at specific values of $V$; in a real disordered sample, there will always be regions where this is true. We add to our original Hamiltonian a small interlayer tunnelling term, which may be written in the form

$$
H_{I L T}=-h \sum_{\mathbf{r}} \sqrt{1-z(\mathbf{r})^{2}} \cos \phi(\mathbf{r}) .
$$

We approximate the $Q$-mode dispersion as

$$
\omega_{Q}(\mathbf{k})=E_{Q 0}+\alpha \mathbf{k}^{2} .
$$

We now consider fluctuations of $\phi(\mathbf{r})$ which consist of two low energy parts, one controlled by the $G$-mode and the other controlled by the $Q$-mode, under the assumption that $T$ is much smaller than the energies of the other modes. We thus write

$$
\phi(\mathbf{r})=\phi_{G}(\mathbf{r})+\phi_{Q}(\mathbf{r}) .
$$

We then integrate out the $Q$-mode to obtain a "renormalized" interlayer tunneling term,

$$
H_{I L T, R}=-h \sum_{\mathbf{r}} \sqrt{1-z(\mathbf{r})^{2}} \cos \phi_{G}(\mathbf{r}) e^{-\left\langle\phi_{Q}(\mathbf{r})^{2}\right\rangle / 2} .
$$

Using Eqs. (39) and (41), we rewrite the last exponential as

$$
\exp \int_{B Z} \frac{d^{2} k}{(2 \pi)^{2}}\left|\psi_{Q}(\mathbf{k}, \mathbf{r}, 2)\right|^{2}\left(n_{Q}(\mathbf{k})+\frac{1}{2}\right) .
$$

For $T \gg E_{Q 0}$ one can approximate the occupation number of the $Q$-mode as

$$
n_{Q}(\mathbf{k}) \approx \frac{T}{E_{Q 0}+\alpha \mathbf{k}^{2}},
$$

so that as $E_{Q 0} \rightarrow 0$, one obtains a logarithmic divergence in the $\mathbf{k}$ integral, provided $\psi_{Q}(\mathbf{k}, \mathbf{r}, 2)$ remains nonzero as $\mathbf{k} \rightarrow 0$. We have verified that indeed it does, leading to a renormalization of $h$ of the form

$$
h_{R} \approx h e^{-T l^{2} \log \frac{\Lambda}{E_{Q 0}} / \alpha} .
$$

Thus, in situations where $E_{Q 0} \rightarrow 0, h$ is strongly suppressed.

\section{B. Suppression of $T_{K T}$}

Recall that at a generic transition in our periodic potential model $E_{Q 0}$ does not vanish. However, as we will now show, the Berezinskii-Kosterlitz-Thouless transition temperature $T_{K T}$ separating the low-temperature, quantum Hall ferromagnet phase from the high-temperature, paramagnetic phase is nevertheless strongly suppressed near transitions. Physically, this is because there are two local minima with nearly the same energy, but different topological density. This makes it very easy for the system to screen vortex/antivortex charge, leading to a very small core $E_{c}$ energy for vortices.

To see this quantitatively, we introduce and analyze an effective, two-dimensional Coulomb gas description of the bilayer quantum Hall ferromagnet. At finite temperature $T$, we approximate the partition function of the quantum Hall ferromagnet $Z \approx \sum_{\{m(\mathbf{R}\}} \exp \left(-E\left[\{m(\mathbf{R}\}] / k_{B} T\right)\right.$ as a sum over the positions of vortices $\{m(\mathbf{R}\}$ with a Coulomb gas energy functional

$$
\begin{gathered}
E\left[\{m(\mathbf{R}\}]=\frac{1}{2} \sum_{\mathbf{R} \neq \mathbf{R}^{\prime}} \mathcal{K}_{s} m(\mathbf{R}) m\left(\mathbf{R}^{\prime}\right) \log \left(\frac{\left|\mathbf{R}-\mathbf{R}^{\prime}\right|}{\xi}\right)\right. \\
-E_{c}(V) \sum_{\mathbf{R}}(m(\mathbf{R}))^{2},
\end{gathered}
$$

where $\mathcal{K}_{s}$ is the bare spin-stiffness computed in Section IIIB $m(\mathbf{R})$ is the vorticity of a plaquette at position $\mathbf{R}$, $\xi$ is an ultraviolet cutoff scale, and $E_{c}$ is the core energy of each vortex/antivortex, whose value depends on $V$, the potential strength, through the potential-dependent details of meron core structure.

To estimate $E_{c}(V)$, we compare the $T=0$ groundstate energy (computed in Section II) of the two competing states whose energies cross at the critical potential strength $V_{c}$. In the simplest case State 1 is a topologically trivial configuration with no vortices, and State 2 


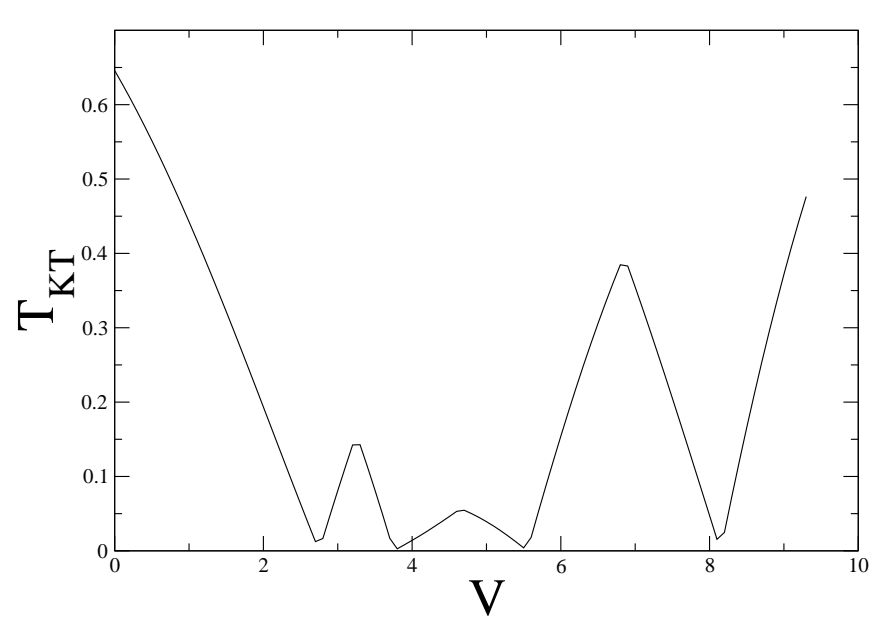

FIG. 10: Finite temperature phase diagram of the bilayer quantum Hall ferromagnet in a periodic potential $V$ (Measured in units of $J$ the nearest-neighbor ferromagnetic exchange energy, using a $16 \ell_{B} \times 16 \ell_{B}$ unit cell with local Coulomb repulsion energy $U=8 J)$. Dramatic decreases in the transition temperature $T_{K T}$ (measured in units of $J / k_{B}$ ) separating the low-temperature ferromagnetic phase from high-temperature paramagnetic phase occur due to changes in the topological density of the ground-state at critical potential strengths.

has two + and two - vortices in a checkerboard pattern in the unit cell. (See, for example, the configuration shown in Fig. 1). Generically, they cross with some slope, with the energy difference per site being

$$
\Delta E=E_{2}-E_{1}=\alpha\left(V-V_{c}\right),
$$

where $V_{c}$ is the transition point. We obtain $\alpha \approx 0.12$ numerically by comparing the energies of the ground and metastable states near $V_{c}$. Without loss of generality, we choose the vortex core size $\xi$ in (47) such that the vortex core energy vanishes as one approaches the transition. Together with (48), this choice implies the following form for the vortex core energy in the vicinity of the transition at $V_{c}$ :

$$
E_{c} \approx \frac{\alpha}{4}\left|V-V_{c}\right|
$$

Note that as one passes through the transition, the high and low energy states interchange roles, and (47) still governs the fluctuations of the system for $V<V_{c}$, with the "absence of a vortex" playing the role of vortices in the free-energy functional. Thus the vortex core energy $E_{c}(V)$ is non-negative across the transition at $V_{c}$.

Following the standard analysis [9] of the twodimensional Coulomb gas (47), we find an implicit equation for $T_{K T}$ in terms of the vortex core energy $E_{c}$ and the ground-state spin stiffness $\mathcal{K}_{s}$

$$
E_{c} \approx k_{B} T_{K T}\left|\ln \left[\frac{1}{\sqrt{2} \pi}\left(1-\frac{2 k_{B} T_{K T}}{\pi \mathcal{K}_{s}}\right)\right]\right| .
$$

We plot in Fig. (10) the result of numerically solving (49) and (50) for $T_{K T}$ as a function of the potential strength $V$. This is one of the central results of this paper. Note that typically, $T_{K T}$ is suppressed by an order of magnitude compared to the Hartree-Fock predictions for a bilayer quantum Hall ferromagnet with no periodic potential.

Near the ground-state transitions, the sharp reduction in $E_{c}$, the core energy for vortex excitations, described by (49), is the principal cause of the dramatic suppression of the low-temperature quantum Hall ferromagnet phase. Far from the ground-state transitions, a secondary effect of the periodic potential on the phase diagram arises through the ground-state spin stiffness $\mathcal{K}_{s}$ : $k_{B} T_{K T} \rightarrow \pi \mathcal{K}_{s} / 2$ for $\left|V-V_{c}\right| \gg 2 \pi \mathcal{K}_{s} / \alpha$. As shown in Fig. 9] the periodic potential reduces $\mathcal{K}_{s}$ dramatically for some values of the potential strength $V$, leading to a suppression of $T_{K T}$ compared to the transition temperature in the absence of a periodic potential.

The Coulomb gas description (47) is expected to overestimate $T_{K T}$ since it contains only a single type of vortex, and focuses on the spin configurations (States 1 and 2 ) with the lowest $T=0$ energy. In the critical regime $V \approx V_{c}$ we observe numerically that the $T=0$ energy of other states approach that of States 1 and 2. Describing the effects of these other states would require generalizing (47) to consider multiple types of vortices. On general grounds, one expects that including more types of vortex fluctuations reduces the finite-temperature stability of the bilayer quantum Hall ferromagnet phase, so the result reported in Fig. 10 is expected to be an upper bound on $T_{K T}$.

\section{CONCLUSIONS, CAVEATS, AND OPEN QUESTIONS}

It has been clear for some time that there are qualitative discrepancies between theoretical predictions for clean systems and the actual phenomenology of $\nu=1$ quantum Hall bilayers at small layer separation. There is a wide consensus in the community that these discrepancies are due to quenched disorder.

Our goal in this paper is to investigate some of the nonperturbative effects of quenched disorder in balanced $\nu=1$ quantum Hall bilayers. Our principal premises are: (i) A strong periodic potential can mimic some of the nonperturbative effects of disorder, and (ii) one needs to focus only on the (pseudo)spin physics. While these premises (especially (i)) can be debated in the context of bilayer quantum Hall systems, there is ample historical evidence of the fruitfulness of (i) in the Bose-Hubbard model and the problem of the quantum Hall plateau transition. Other limitations of this work include the neglect of long-range Coulomb interactions between induced topological densities, the neglect of the renormalization of the classical ground states we found due to fluctuations, the neglect of interactions between the lowlying modes, especially the $G$ - and $Q$-modes, and the perturbative treatment of the interlayer tunneling. We 
see this work as a first step in a systematic investigation of these effects.

Starting from the (rather minimal) model Hamiltonian, Eq. (3), we study the ground states and the collective excitations numerically for interlayer tunneling $h=0$. As the strength of the periodic potential increases, we observe generically first-order transitions between states with different topological densities. Occasionally, these transitions are weakly first-order, with a new, charge-carrying, quadratically dispersing mode becoming nearly gapless at the transition. Such a mode can suppress the interlayer tunneling amplitude strongly at nonzero $T$. Even when the transitions are strongly firstorder, we show that vortices become very easy to create, and drive the Berezinskii-Kosterlitz-Thouless transition temperature to zero at the transition.

It is important to note that there is a qualitative difference between weak and strong $h$ in our approach. While our numerics are carried out only for $h=0$, the inclusion of an interlayer tunneling amplitude much smaller than any other energy scale will not affect any of the qualitative physics we uncover. However, if the strength of the interlayer tunneling $h$ is increased while other parameters such as $E_{c}$ and the strength of the periodic potential $V$ are kept fixed, we expect the system to undergo a set of ground state phase transitions leading ultimately to the uniform ferromagnetic ground state at very large $h$. This qualitative distinction between weak and strong tunneling does not exist in the clean model[9], but is consistent with experiments.

Based on our results for the periodic potential, we can speculate about the effects of true quenched disorder. There are several important effects: Firstly, it can be shown via a mapping to the random field Ising model 56] that disorder converts the sequence of firstorder transitions we found as $V$ increases into a sequence of second-order transitions. While this mapping is rigorously provable only for classical models, it is believed to hold for many quantum phase transitions as well [57, 58] (the exceptions seem to be transitions that are completely smeared out and destroyed by disorder [59]). This sequence of second-order transitions maintains the qualitative distinction between weak and strong tunneling mentioned earlier. Furthermore, at the quantum level, this implies that the $Q$-mode generically becomes gapless at ground state transitions with true disorder. Secondly, even away from a ground-state transition, in a system with quenched disorder there will be large regions which are close to a transition (the system is in a Griffiths phase [60]). Being in a Griffiths phase also means that excitations of arbitrarily low energy are available fromlarge rare regions close to the transition, which leads to divergent low-frequency susceptibilities throughout the Griffiths phase 61] at $T=0$. For $T \neq 0$, since vortices are easy to create in such regions, $T_{K T}$ is expected to be strongly suppressed throughout the sample. This is consistent with the fact that no low-temperature phase with the phenomenology of the BKT power-law phase (includ- ing a Josephson-like delta-function peak in the interlayer conductance) has yet been observed in experiments. This suggests two possibilities: (i) The true ground state of the system at $T=0$ is ferromagnetically ordered, but current experiments have probed only the $T>T_{K T}$ regime. (ii) The true ground state is quantum disordered due to some combination of quantum fluctuations/quenched disorder.

Even in the more conventional possibility (i), there are several natural sources for the dissipation seen in experiments. The dissipation could be due to unbound vortices in the hydrodynamic transport regime 62 ] $\hbar \omega k_{b} T$, and/or due to the low-energy Griffiths $Q$-modes.

A second aspect of disorder is that as one crosses domains of different topological density, the spin wave velocity varies sharply. This is expected to lead to chaotic reflections of spin waves leading to a diffusive-like behavior at macroscopic length-scales 63.

Yet another aspect concerns the critical counterflow velocity, which determines the critical counterflow current. In regions where the $Q$-mode has a very small gap, the critical velocity will also be small, and nonlinear/dissipative effects 25] will be visible at very tiny counterflow current. Similar physics holds for the critical interlayer tunneling current density. Some recent work takes the point of view that perhaps the experiments are in the $T<T_{K T}$ regime, but the smallness of the critical tunneling current density is a primary mechanism driving the observed dissipation [43].

Let us now turn to issues of zero-temperature physics. It is possible fluctuations quantum disorder the ground state (even in the periodic-potential model) near the mean-field ground state transitions that we found. Clearly, one needs an effective theory of the low-lying modes and their interactions to address these issues. An important source of quantum disordering is the tunneling of (multiple) vortices. Due to the spin-charge relation, the quantum tunneling events (called hedgehogs) of a single vortex will violate charge conservation. However, especially near transitions, one can imagine multiple vortices in a unit cell with total vorticity zero tunneling together. This has a connection to ideas of deconfined criticality 64 66. In this type of scenario, first described for quantum dimer 64] models and quantum antiferromagnets 65], dimers/spin excitations, are described as composites of monomers/spinons. Single hedgehog events are forbidden due to lattice symmetries, but multiple hedgehogs are allowed. In certain well-defined extentions of quantum antiferromagnets, the multiple-hedgehog events can be shown to be irrelevant 67], leading to a critical point with deconfined spinons 66].

The important difference is that in the picture of the quantum Hall bilayer presented here, it is not lattice symmetries but the spin-charge relation [52, 53] which enforces the absence of single hedgehog events. Thus, if deconfined criticality were to occur for the periodic potential model, it would also likely occur in the model with true disorder. 
The quantum Hall bilayer remains a rich system which potentially supports a host of physical behaviors yet to be explored, particularly in the presence of strong disorder potentials that are almost certainly a feature of their realization in semiconductor systems. We believe immersing the system in a periodic potential offers a window through which one may begin an exploration of this physics.
GM wishes to thank Leon Balents for illuminating conversations, and also the Kavli Institute for Theoretical Physics at Santa Barbara and the Aspen Center for Physics for their hospitality when some of this work was carried out. JS and HAF are grateful to the NSF for partial support under DMR-0704033, while GM and NB-A wish to thank the NSF for partial support under DMR0703992 .
[1] L. Keldysh and Y. Kopaev, Sov. Phys. J. 6, 2219 (1965).

[2] L. V. Butov, C. W. Lai, A. L. Ivanov, A. C. Gossard, and D. S. Chemla, Nature 417, 47 (2002); D. Snoke, Science 298, 1368 (2002).

[3] See J.P. Eisenstein and A.H. MacDonald, Nature 432, 691 (2004), and references therein.

[4] N. Read and S. Sachdev, Phys. Rev. Lett. 75, 3509 (1995); C. Timm, S. M. Girvin, P. Henelius, and A. V. Sandvik, Phys. Rev. B 58, 1464 (1998).

[5] H.A. Fertig, Phys. Rev. B 40, 1087 (1989).

[6] X.-G. Wen and A. Zee, Phys. Rev. Lett. 69, 1811 (1992); Phys. Rev. B. 47, 2265 (1993); Z. F. Ezawa and A. Iwazaki, Phys. Rev. B 47, 7295 (1993).

[7] K.Moon, H.Mori, K.Yang, S.M.Girvin, A.H.MacDonald, L.Zheng, D.Yoshioka, and S.-C.Zhang, Phys. Rev. B 51, 5138 (1995); K.Yang, K.Moon, L.Belkhir, H.Mori, S.M.Girvin, A.H.MacDonald, L.Zheng, and D.Yoshioka, Phys. Rev. B 54, 11644 (1996).

[8] V. L. Berezinskii, Sov. Phys. JETP 34, 610 (1971); J. M. Kosterlitz and D. J. Thouless, Jour. Phys. C6, 1181 (1973).

[9] J. V. Jose, L. P. Kadanoff, S. Kirkpatrick, and D. R. Nelson, Phys. Rev. B 16, 1217 (1977).

[10] S.M.Girvin and A.H.MacDonald, Chapter 5 of Perspectives in Quantum Hall Effects, Das Sarma and Pinczuk, Editors, 1997.

[11] G. S. Boebinger, H. W. Jiang, L. N. Pfeiffer, andK. W. West, Phys. Rev. Lett. 64, 1793 (1990); A. H. MacDonald, P. M. Platzman, and G. S. Boebinger, Phys. Rev. Lett. 65, 775 (1990); Y. W. Suen, J. Jo, M. B. Santos, L. W. Engel, S. W. Hwang, and M Shayegan, Phys. Rev. B 44, 5947 (1991); S. Q. Murphy, J. P. Eisenstein, G. S. Boebinger, L. N. Pfeiffer, and K. W. West, Phys. Rev. Lett. 72, 728 (1994).

[12] J. K. Jain, Phys. Rev. Lett. 63, 199 (1989); Phys. Rev. B 41, 7635 (1990; Science 266, 1199 (1994).

[13] B. I. Halperin, P. A. Lee, and N. Read, Phys. Rev. B 47, 7312 (1993).

[14] I. B. Spielman, J. P. Eisenstein, L. N. Pfeiffer, and K. W. West, Phys. Rev. Lett. 84, 5808 (2000); M. Kellogg, I. B. Spielman, J. P. Eisenstein, L. N. Pfeiffer, and K. W. West, Phys. Rev. Lett. 88, 126804 (2002).

[15] L. Tiemann, W. Dietsche, M. Hauser, and K. von Klitzing, New Jour. Phys.10, 045018 (2008).

[16] I. B. Spielman, J. P. Eisenstein, L. N. Pfeiffer, and K. W. West, Phys. Rev. Lett. 87, 036803 (2001).

[17] M. Kellogg, J.P. Eisenstein, L.N. Pfeiffer, and K.W. West, Phys. Rev. Lett. 93, 036801 (2004); E. Tutuc, M. Shayegan, and D. Huse, Phys. Rev. Lett. 93, 036802 (2004).

[18] A. D. K. Finck, A. R. Champagne, J. P. Eisenstein, L.
N. Pfeiffer, and K. W. West, Phys. Rev. B 78, 075302 (2008).

[19] L. Tiemann, Y. Yoon, W. Dietsche, K. von Klitzing, and W. Wegscheider, Phys. Rev. B 80, 165120 (2009).

[20] It should be noted that even below the "critical" interlayer tunnelling current the $I-V$ curve has a nonzero slope in the measurements of Ref. 19]. It is not clear that the quantity being measured is the analog of a critical current in a Josephson junction.

[21] A. R. Champagne, J. P. Eisenstein, L. N. Pfeiffer, and K. W. West, Phys. Rev. Lett. 100, 096801 (2008).

[22] M. Stone, Phys. Rev. B 53, 16573 (1996).

[23] L. Balents and L. Radzihovsky, Phys. Rev. Lett. 86, 1825 (2001).

[24] A. Stern, S.M. Girvin, A.H. MacDonald, and Ning Ma, Phys. Rev. Lett. 86, 1829 (2001).

[25] M. M. Fogler and F. Wilczek, Phys. Rev. Lett. 86, 1833 (2001).

[26] Y. N. Joglekar and A. H. MacDonald, Phys. Rev. Lett. 87, 196802 (2001); R. L. Jack, D. K. K. Lee, adn N. R. Cooper, Phys. Rev. B 71, 085310 (2005).

[27] E. Demler, C. Nayak, and S. Das Sarma, Phys. Rev. Lett. 86, 1853 (2001); Y.-B. Kim, C. Nayak, E. Demler, N. Read, and S. Das Sarma, Phys. Rev. B 63, 205315 (2001).

[28] A. A. Burkov and A. H. MacDonald, Phys. Rev. B 66, 115320 (2002).

[29] H. A. Fertig, Phys. Rev. Lett. 89, 035703 (2002)

[30] H. A. Fertig and J. P. Straley, Phys. Rev. Lett. 91, 046806 (2003).

[31] S. Rapsch, J. T. Chalker, and D. K. K. Lee, Phys. Rev. Lett. 88, 036801 (2002); D. K. K. Lee, S. Rapsch, and J. T. Chalker, Phys. Rev. B 67, 195322 (2003).

[32] M. Abolfath, A. H. MacDonald, and L. Radzhihovski, Phys. Rev. B 68, 155318 (2003).

[33] D. Sheng, L. Balents, and Z. Wang, Phys. Rev. Lett. 91, 116802 (2003).

[34] N. E. Bonesteel, I. A. McDonald, and C. Nayak, Phys. Rev. Lett. 77, 3009 (1996); S. H. Simon, E. H. Rezayi, and M. V. Milovanovic, Phys. Rev. Lett. 91, 046803 (2003).

[35] R. Rajaraman, Phys. Rev. B 56, 6788 (1997); T. Morinari, Phys. Rev. B 59, 7320 (1999); J. Ye, cond-mat/0310512, (2003); G. S. Jeon and J. Ye, Phys. Rev. B 71, 035348 (2005).

[36] Z. Wang, Phys. Rev. Lett. 92, 136803 (2004); Phys. Rev. Lett. 94, 176804 (2005).

[37] D.A. Huse, cond-mat/0407452 (2004).

[38] J. Schliemann, S. M. Girvin, and A. H. MacDonald, Phys. Rev. Lett. 86, 1849 (2001); K. Yang, Phys. Rev. Lett. 87, 056802 (2001); M. Abolfath, L. Radzihovsky and A. H. MacDonald, Phys. Rev. B 65, 233306 (2002); K. Nomura 
and D. Yoshioka, Phys. Rev. B 66, 153310 (2002); J Schliemann, Phys. Rev. B 67, 035328 (2003).

[39] H. A. Fertig and G. Murthy, Phys. Rev. Lett. 95, 156802 (2005); Sol. State. Commun. 140, 83 (2006).

[40] B. Roostaei, K. J. Mullen, H. A. Fertig, and S. H. Simon, Phys. Rev. Lett. 101, 046804 (2008).

[41] G. Murthy and S. Sachdev, Phys. Rev. Lett. 101, 226801 (2008).

[42] P. R. Eastham, N. R. Cooper, and D. K. K. Lee, Phys. Rev. B 80, 045302 (2009).

[43] J.-J. Su and A. H. MacDonald, Nature Physics 4, 799 (2008); arXiv:1001.2923 [cond-mat.mes-hall].

[44] A. L. Efros, Sol. State. Commun. 65, 1281 (1988); Sol. State. Commun. 70, 253 (1989); Phys. Rev. B 45, 11354 (1992); A. L. Efros, F. G. Pikus, and V. G. Burnett, Phys. Rev. B 47, 2233 (1993); F. G. Pikus and A. L. Efros, Phys. Rev. B 47, 16395 (1993).

[45] S. Ilani, J. Martin, E. Teitelbaum, J. H. Smet, D. Mahalu, V. Umansky, and A. Yacoby, Nature 427, 328 (2004).

[46] M. P. A. Fisher, P. B. Weichmann, G. Grinstein, and D. S. Fisher, Phys. Rev. B 40, 546 (1989).

[47] W. Chen, M. P. A. Fisher, and Y.-S. Wu, Phys. Rev. B 48, 13749 (1993); X.-G. Wen and Y.-S. Wu, Phys. Rev. Lett. 70, 1501 (1993); L. P. Pryadko and S.-C. Zhang, Phys. Rev. B 54, 4953 (1996); J. Ye and S. Sachdev, Phys. Rev. Lett. 80, 5409 (1989).

[48] A. W. W. Ludwig, M. P. A. Fisher, R. Shankar, and G. Grinstein, Phys. Rev. B 50, 7526 (1994).

[49] I. B. Spielman, L. A. Tracy, J. P. Eisenstein, L. N. Pfeiffer, and K. W. West, Phys. Rev. Lett. bf 94, 076803 (2005); N. Kumada, K. Muraki, K. Hashimoto, and Y. Hirayama, Phys. Rev. Lett. 94, 096802 (2005); S. Luin, V. Pellegrini, A. Pinczuk, B. S. Dennis, L. N. Pfeiffer, and K. W. West, Phys. Rev. Lett. 94, 146804 (2005).

[50] Z. F. Ezawa, Phys. Rev. Lett. 82, 3512 (1999); S. Ghosh and R. Rajaraman, Phys. Rev. B 63, 035304 (2001); Z. F. Ezawa and K. Hasebe, Phys. Rev. B 65, 075311 (2002), ; Z. F. Ezawa and G. Tsitsishvili, Phys. Rev. B 70, 125304 (2004); J. Bourassa, B. Roostaei, R. Coté, H. A. Fertig, and K. Mullen, cond-mat/0607784

[51] P. Guidici, K. Muraki, N. Kumada, Y. Hirayama, and T. Fujikawa, Phys. Rev. Lett. 100, 106803 (2008); A. D. K.
Finck, J. P. Eisenstein, L. N. Pfeiffer, and K. W. West, Phys. Rev. Lett. 104, 016801 (2010).

[52] D.-H. Lee and C. L. Kane, Phys. Rev. Lett. 64, 1313 (1990).

[53] S. L. Sondhi, A. Karlhede, S. A. Kivelson, and E. H. Rezayi, Phys. Rev. B 47, 16419 (1993); H. A. Fertig, L. Brey, R. Cote, A. H. MacDonald, A. Karlhede, S. L. Sondhi, Phys. Rev. B 55, 10671 (1997).

[54] S. Sachdev and K. Park, Annals of Phys. 298, 58 (2002).

[55] F. D. M. Haldane, Phys. Rev. Lett. 61, 1029 (1988).

[56] Y. Imry and S.-K. Ma, Phys. Rev. Lett. 35, 1399 (1975); M. Aizenman and J. Wehr, Phys. Rev. Lett. 62, 2503 (1989); K. Hui and A. N. Berker, Phys. Rev. Lett. 62, 2507 (1989).

[57] R. Shankar and G. Murthy, Phys. Rev. B 36, 536 (1987); D. S. Fisher, Phys. Rev. Lett. 69, 534 (1992); Phys. Rev. B 51, 6411 (1995).

[58] S. Sachdev, Quantum Phase Transitions, Cambridge University Press, New York, 2001.

[59] J. A. Hoyos and T. Vojta, Phys. Rev. Lett. 100, 240601 (2008); D. J. Schwab and S. Chakravarty, Phys. Rev. B 79, 125102 (2009).

[60] R. B. Griffiths, Phys. Rev. Lett. 23, 17 (1969).

[61] M. J. Thill and D. A. Huse, Physica (Amsterdam), 214A, 321 (1995); A. P. Young and H. Reiger, Phys. Rev. B 53, 8486 (1996); M. Guo, R. N. Bhatt, and D. A. Huse, Phys. Rev. B 54, 3336 (1996).

[62] K. Damle and S. Sachdev, Phys. Rev. B 56, 8714 (1997); S. Sachdev, Phys. Rev. B 59, 14054 (1999).

[63] S. John, H. Sompolinski, and M. J. Stephen, Phys. Rev. B 27, 5592 (1983); S. John and M. J. Stephen, Phys. Rev. B 28, 6358 (1983).

[64] E. Frakdin, D. A. Huse, R. Moessner, V. Oganesyan, and S. L. Sondhi, Phys. Rev. B 69, 224415 (2004).

[65] A. Vishwanath, L. Balents, and T. Senthil, Phys. Rev. B 69, 224416 (2004).

[66] T. Senthil, L. Balents, S. Sachdev, A. Vishwanath, and M. P. A. Fisher, Science 303, 1490 (2004); Phys. Rev. B 70, 14407 (2004).

[67] G. Murthy and S. Sachdev, Nucl. Phys. B334, 557 (1990). 